\title{
IMPACT OF SIGNAL RADIATION LEAKAGE ON POWER LINE COMMUNICATION SYSTEMS
}

\author{
Ganiyu Adedayo Ajenikoko ${ }^{1}$ and Ezekiel Olusegun Ojekunle ${ }^{2}$ \\ 1,2. Department of Electronic and Electrical Engineering, Ladoke Akintola University of Technology, P.M.B, \\ 4000, Ogbomoso, Nigeria. \\ Corresponding Email: ajeedollar@gmail.com
}

\begin{abstract}
Power Line Communication (PLC) is the usage of electrical power supply networks as a transmission medium for the transfer of various telecommunication services. However, PLC associated with the major cause of harmful interference (radiation leakage) to other users in transmission of information affects the full development and success of PLC. There is the need to develop a proper standard and regulations that will eliminate this problem globally. This study therefore, studies the impact of signal radiation leakage on Power Line Communication systems using Numerical Electromagnetic Code (NEC) method and field measurement to measure spacing along the power line and for measuring distance between the system frequencies in the power line. The result shows that the spacing between the wires on the power lines has effect on the radiation leakage. As wire spacing increases and external cancellation degrades, radiation escapes similar to those of long wire antennas. The result also shows that the frequencies of power line operation lies between $444 \mathrm{kHz}$ and $482 \mathrm{kHz}$. In general the PLC electric field along and near the power line decay with distance from the device but in at least one case, the electric field actually increased with increasing distance from the PLC device. Therefore, high levels of radiation suppression can be achieved only if the wires are very close together in terms of wavelength and balanced signals.
\end{abstract}

Keywords: Power Line Communication(PLC) , Numerical Electromagnetic Code (NEC), Field Measurement, Electrical Power Network, Radiation Leakage, Frequency.

DOI: $10.7176 / \mathrm{MTM} / 9-8-02$

Publication date: August 31st 2019

\subsection{Introduction}

Power Line Communication (PLC) is a recent and rapidly growing technology, which aims at the electricity of the power lines for transmission of data, to get to the end user and enable them to access all data. It can be considered as attractive system because it can reach more homes than coaxial cable systems or telephone lines [1]. PLC can provide cost effective connectivity solution. PLC could be a long distance, medium and low transmission. In PLC, an Electric Automation Meter (EMR) reading can be installed to read the total energy consumption by a consumer. In PLC, broadband power line provide benefits over regular cable connections: the extensive infrastructure already available appears to allow people in remote locations to access the internet with relatively little equipment by the utility $[2,3]$.

The greatest advantage of power lines as communication media include accessibility to customers. Power utilities have been using this technology for many years to send or receive data on the power grid using the existing infrastructure. In power line communication, transmission of voltage is from generating station to transmitting station followed by distribution to local transformer where consumers may be fed, that is electrical energy is transmitted over a high voltage, distributed over medium voltage and then lower voltage used 
by consumers(end user). Every consumer must be able to access all data and service at any time and any place in the home, regardless of where their gadget is placed $[2,4,5]$.

Power Line Communication or Power Line Carrier (PLC), also known as Power Line Digital Subscriber Line (PDSL), Power Line Telecom (PLT), or Power Line Networking (PLN), or Broadband over Power Lines (BPL) are systems for carrying data on a conductor. In the frequency band near to the power frequency $(50 \mathrm{~Hz})$, PLC systems do not experience as much hostility as they would at frequencies greater than that of the spectrum. Unfortunately, at low frequencies only limited data rates can be achieved since frequency is the sole controlling element of bandwidth $[1,4,6]$. The demand for broadband services dictates that higher frequency bands are used and as frequency increases, so the complications [2,6].

PLC could either be in long distance, medium, or low transmission and reception. The PLC is a communication medium between the transmitter and the receiver which is known as power line channel or powerline carrier modem . Data rates over a power line communication system vary widely [2]. Low-frequency (about 100-200 KHz) carriers impressed on highvoltage transmission lines may carry one or two analog voice circuits, or telemetry and control circuits with an equivalent data rate of a few hundred bits per second; moreover, the circuits may be many miles long. Higher data rates generally imply shorter ranges; a local area network operating at millions of bits per second may only cover one floor of an office building, but eliminates installation of dedicated network cabling [5, 7, 8].

PLC technologies, measurement modeling and characterization, as well as improvement techniques in performance of PLC systems is at the physical and link layers that need attention. There are many number of technical contributions providing understanding how PLC systems operate and pointing out challenging advancements related to on PLC technology. Coupling antenna was the first type of coupling used with the electric power grid due to its simplicity, easy installation and cost $[1,8,9]$.

Furthemore, mismatching of impedance with the power cables, which reduces the transfer of energy between the power cable and the antenna, motivated investigations which resulted in the introduction of capacitive couplers [4]. However, some researchers have reinvestigated the use of antennas to introduce PLC wireless system. On the other hand, their usage in electric power systems Low-Voltage (LV) was not widely adopted due to the high insertion loss. For LV levels, capacitive coupling is common due to its low cost and simplicity. Most recently, resistive PLC couplers have gained attenutions due to their low cost and simple circuitry features $[7,9,10]$.

The PLC modem design is a challenging problem, particularly in coping with the various sources of radiation leakage and frequencies [7]. As a result, this study is aimed to present Numerical Electromagnetic Code (NEC) and field measurement techniques for mitigating the signal radiation and frequencies in PLC systems. These techniques will deal with PLC channel conditions and to achieve higher data rates that will reduce the signal radiation in PLC system.

\subsection{PLC Impairments}

Power line as a communication medium presents inherent impairments that a designer has to accept their impact as a base loss [11]. Besides these inherent impairments, the very systems that the electrical utility sort to supply electricity, consume considerable amounts of the transmission signal and presents a harsh environment by feeding harmonics and noise back into the grid randomly is very significant. The challenge of designing PLC systems stems from these key issues. The intrinsic properties of the electrical wiring have a direct influence on the attenuation level at different frequencies in the 1-30 MHz frequency band. Transmission with low frequency modulation $(\sim 100 \mathrm{kHz})$ will result in low attenuation as 
compared to transmission at $\sim 10 \mathrm{MHz}$. This is due to the skin effect which causes about 10 times smaller effective conductor area since the frequency is higher by a factor of $100[8,12$, 13].

However, due to the self-inductance of the conductor, the resultant conductor impedance is proportional to the transmission frequency. The higher the frequency, the more difficult it becomes for the signal to transverse the wiring [12, 14]. Moreover, power devices and other loads have an adverse effect on the low frequency systems since these inductive loads and particularly resistive loads conduct very well at low frequency thereby draining the energy from the communication signal. Conversely, the R-L loads have lesser effect on the high frequency systems as inductive loads permit an insignificant amount of the high frequency current to pass through. Capacitive and numerous protective devices such as varactors and zener diodes are however obstacles to transmission of high frequency signals [2, $13,15]$.

\subsection{Interference in PLC}

Electrical signals travelling along a cable causes some radiation of the signals into the surrounding. These radiated signals interfere with nearby electronic equipment, resulting in malfunctioning of such equipment [16]. This is referred to as Electromagnetic Interference (EMI). Other cable types - coaxial, optic-fibre, twisted-pair cables-by design are meant to transmit data signal while limiting potential antenna effects, power line cables inherently tend to act as unintentional antennas. Hence, when the power line network is used to transmit radio frequency (RF) signals (in the range of $10 \mathrm{kHz}-30 \mathrm{MHz}$ ), some of these signals may leak, interfering with signals from other devices that are connected on the PLC network. This results in undesirable degradation of transmitted signals and reduction in throughput; some of the connected appliances may also inject noise into power line cables, contributing further to performance degradation of the PLC network [17, 18, 19].

RF signals leaking from several devices on the PLC network in nearby buildings may combine to form a composite signal significant enough to interfere with, and distort signals transmitted over these channels [18]. Unfortunately, most of the regulations on PLC are restricted to a single device under monitoring and testing. In a widespread PLC scenario, where numerous sources are unintentionally radiating in parallel, the wire structures that transmit the PLC signals form an antenna array thereby contributing to the far field. In addition, certain portion of the transmitted power are radiated through ground and sky wave, affecting highly sensitive short wave radio services like amateur radios, wireless security services, and military surveillance stations. Concisely, when PLC signals overlay frequency ranges of wireless services, occurrence of interference becomes unavoidable; the extent of this interference depends largely on the transmission power and distance, as well as on the structural layout of wires $[19,20]$.

For a common-mode cable pair, current signals flow in the same direction on both conductors, where the return portions follow the ground path. Differential mode on the other hand, ensures that current signals flow in opposite directions on the cable pair. Thus, a highly symmetrical line implies a large ratio of differential to common mode current flow, and by extension, very weak radiation, while a nonsymmetrical line results in common-mode current flow, inducing high radiation $[10,21,22]$.

\subsection{Mitigating Radiation Effects in PLC}

A wireless signal transmitting on short-wave, high-frequency spectrum are susceptible to interference and in the worst case, blockage from PLC signals. In view of this, it is highly 
expedient to minimize radiation and unwanted radiation from PLC. In order to minimize the radiation effect in PLC, the following steps are recommended [3, 9, 22]:

i. Incorporation of network conditioners into PLC adapters or networks. These will help reduce radiation significantly by maintaining the symmetrical balance in the power line cables.

ii. Installing high-frequency filters at power line ends. This keeps the PLC signals on the intended propagation paths and also prevents them from entering attached devices or conductors with high radiation efficiency.

iii. Using appropriate power supply cable configuration that exploits the "natural" symmetry inherent in certain configurations.

iv. Reducing the power spectral density of PLC signals: Since PLC signal emissions are measured within a limited bandwidth, shrinking the power spectral density immediately

lowers the radiation levels, while maintaining the same transmitted power.

\subsection{Materials and Method.}

In this work Numerical Electromagnetic Code (NEC) and Field measurement technique were used to study the impact of signal radiation leakage on power line communication system. The Numerical Electromagnetic Code, NEC is a user oriented computer code for the analysis of the electromagnetic response of antennas and other metal structures. It is built around the numerical solution of integral equations for the currents induced on the structure by sources or incident fields. The approach avoids many of the simplifying assumptions required by other solution methods and provides a highly accurate and versatile tool for electromagnetic analysis.

The field measurements of PLC signal power at locations along and near an energized line and PLC signal power at various distances away from an energized line were conducted. The measurements were made using an RF spectrum analyzer positioned two meters above the ground.

\subsection{Modelling of Power Lines using Numerical Electromagnetic Code}

The NEC software program was used to create a number of power line models to gain a greater understanding of the impact of signal radiation leakage on power line communication system. The arrangement of medium voltage power line consists of three horizontal parallel aluminum conductor wires which are about $12 \mathrm{~mm}$ in diameter and $10 \mathrm{~m}$ above the ground. The conductors have a minimum line spacing of about $400 \mathrm{~mm}$, and that of a three phase low voltage, power line consisting of four vertical parallel wires which are about $10 \mathrm{~mm}$ in diameter and $7 \mathrm{~m}$ above the ground.

To examine the effect of increasing power line wire spacing on radiation leakage using the NEC model described above, the resistance of the conductors was assumed to be zero. This study considered only the balanced mode configuration, as this is the only mode recognized as being capable of significant external field cancellation. The values of the source and load powers are obtained as a function of frequency and load impedance. For the fact that the line is located in free space and has no resistance, the only loss mechanism for the line is through radiation. That is, the difference between the source power $P_{S r C}$ and the load power $P_{\text {load }}$ must be completely due to radiation. Hence, the percentage power radiated by the line $P_{\text {rad }}$ is calculated as: 


$$
P_{\text {rad }}=100\left[1-\frac{P_{\text {Load }}}{P_{s r c}}\right]
$$

\subsection{Modelling of Power Lines using Field Measurement}

Measurements were performed with a goal of quantifying key aspects of PLC signals. The measurements were conducted at the VRA substation where PLC system is currently deployed for line protection and voice communication. The measurements were conducted using PLC signal power at locations along and near an energized line. PLC signal power at various distances away from an energized line and measurements were made using an RF spectrum analyzer positioned two meters above the ground.

\subsection{Discussion of Results.}

The analysis of results for the signal radiation leakage on power line communication system is presented in Figure 1 to Figure 3.

The effect of power line wire spacing on PLC signal radiation leakage is shown in Figure 1. Figure 1 shows how the radiated power, $P_{r a d}$ varies from 20 to $80 \mathrm{MHz}$ on a line spacing of $0.5 \mathrm{~m}, 1 \mathrm{~m}, 1.5 \mathrm{~m}$ and $2 \mathrm{~m}$. The radiated power increases significantly with increasing frequency because the spacing between the line conductors is larger compared to the wavelength as the frequency and line spacing increases. The maximum value shown is $70 \%$ of input power for the case of $80 \mathrm{MHz}$ on $2 \mathrm{~m}$ spacing line. This is due to the fact that $2 \mathrm{~m}$ is considered to be $53.3 \%$ of a wavelength at $80 \mathrm{MHz}$. However, the system did not improve very quickly as frequency lowers and the line spacing is halved. At $1 \mathrm{~m}$ spacing and $20 \mathrm{MHz}$, the radiation is still about $2 \%$ of the input power.

In addition, the spacing between the wires on the power lines has effect on the radiation leakage. As wire spacing increases and external cancellation degrades, radiation escapes similar to those of long wire antennas. External cancellation of equal and opposite fields relies on the signals on the two wires being of opposite sign, equal in amplitude, and physically closed together in terms of wavelength. High levels of radiation suppression can be achieved only if the wires are very close together in terms of wavelength and carry carefully balanced signals.

For the field measuremt technique, the measurement results for PLC signal radiation along and away from the power line are given in Figures 2 and 3 respectively. Figure 2 shows the distance along the power line at frequencies of $444 \mathrm{kHz}$ and $482 \mathrm{kHz}$. Figure 3 indicates the distance away from power line at frequencies $444 \mathrm{kHz}$ and $482 \mathrm{kHz}$. The result shows that the frequencies of operation lie between $444 \mathrm{kHz}$ and $482 \mathrm{kHz}$. These measurements indicate that, in general the PLC electric field along and near the power line decay with distance from the device. In at least one case, the electric field actually increased with increasing distance from the PLC device. This is due to PLC signal reflection by one or more impedance discocontinuities and the generation of standing waves.

In general, the location variability in the field strength is due to the presence of standing waves in the current distribution along the power line. The measurement results of PLC signal power at the distance away from the PLC device indicates that there is a decrease in received power with an increase in distance from the PLC device and power line. The measurement results of PLC signal power at some distances along the power line is at a frequency range of $444 \mathrm{kHz}$ and $482 \mathrm{kHz}$. 


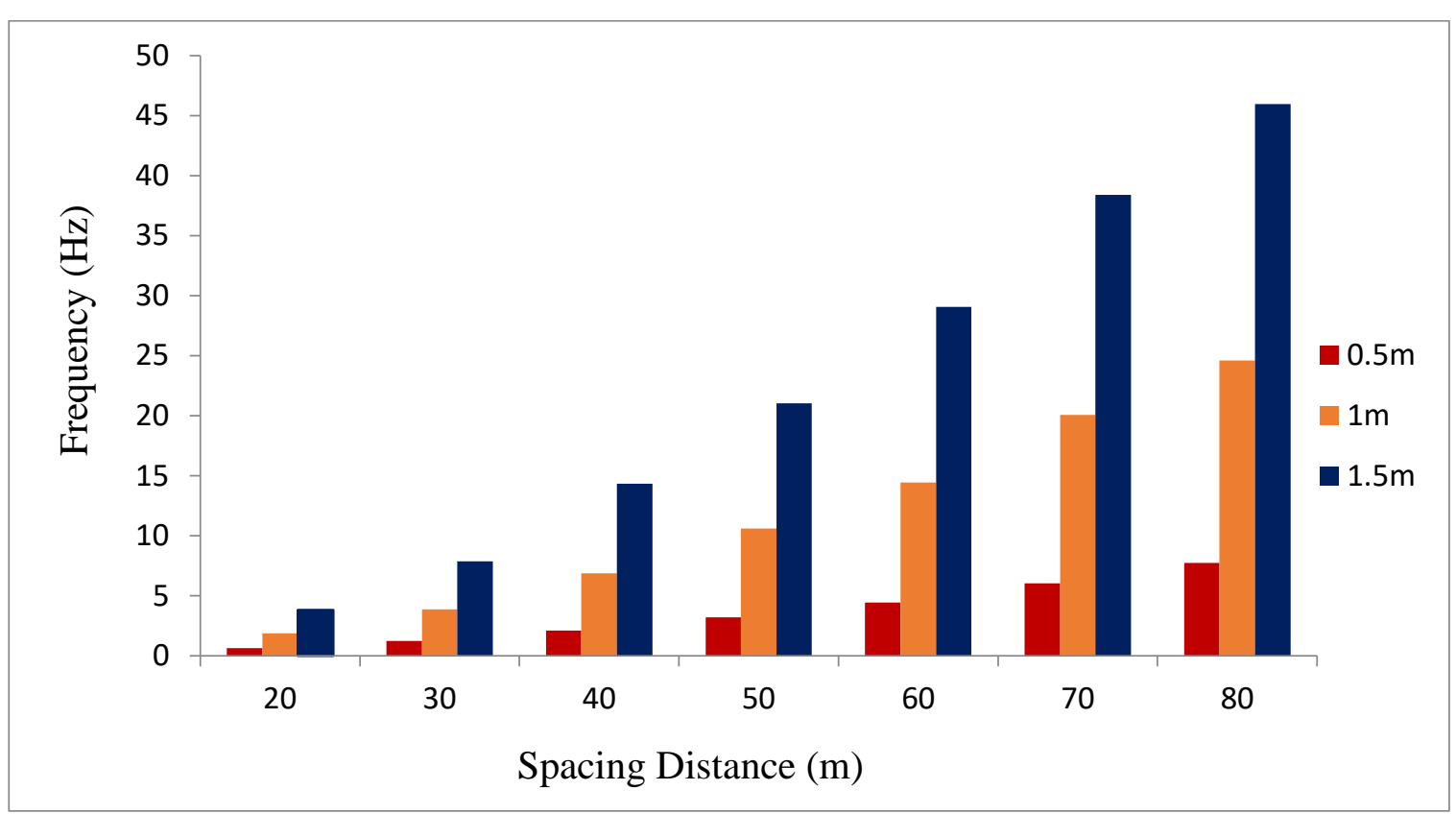

Figure 1: Effect of power line wire spacing distance on PLC signal radiation leakage

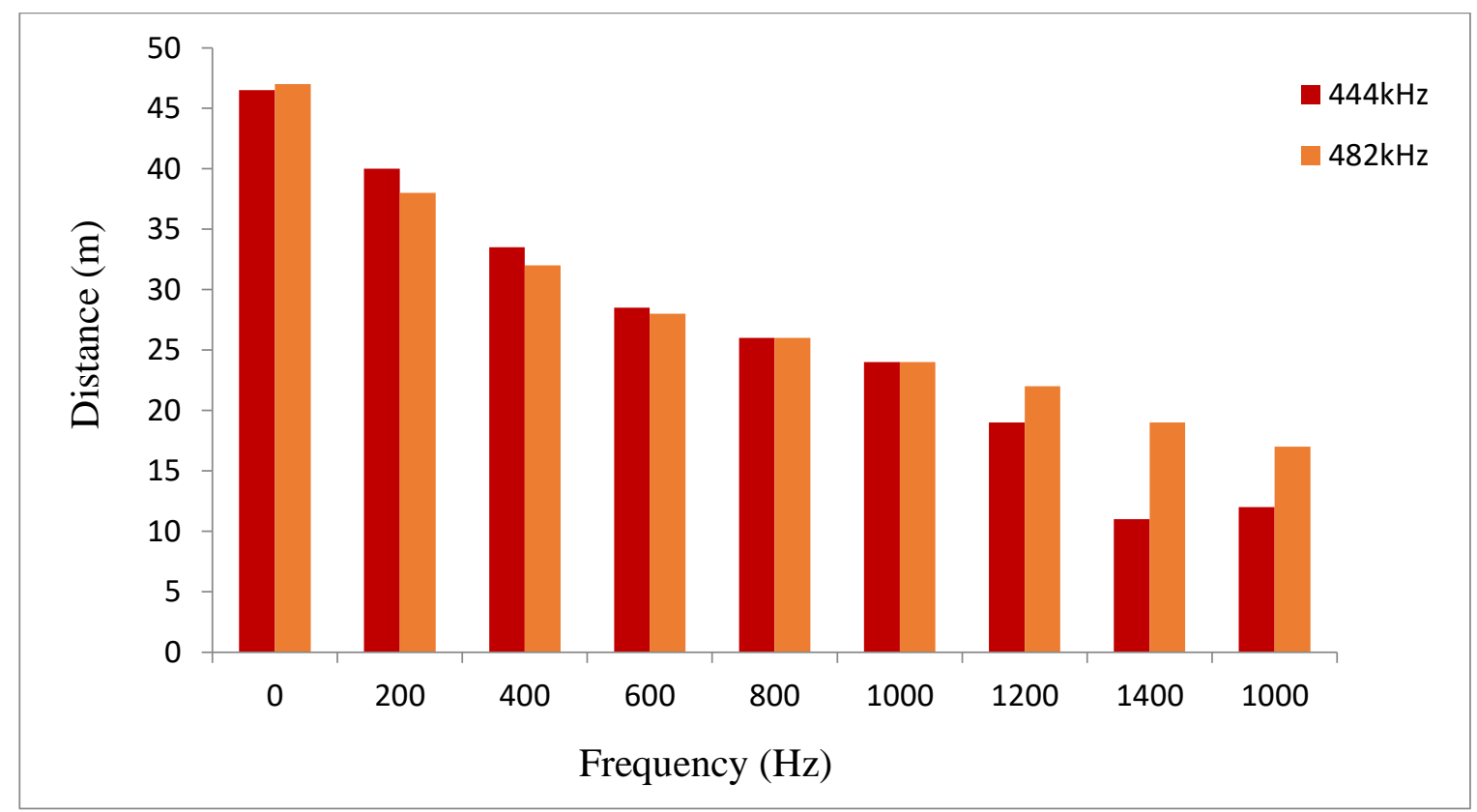

Figure 2: Distance along the power line at frequencies of $444 \mathrm{kHz}$ and $482 \mathrm{kHz}$ 


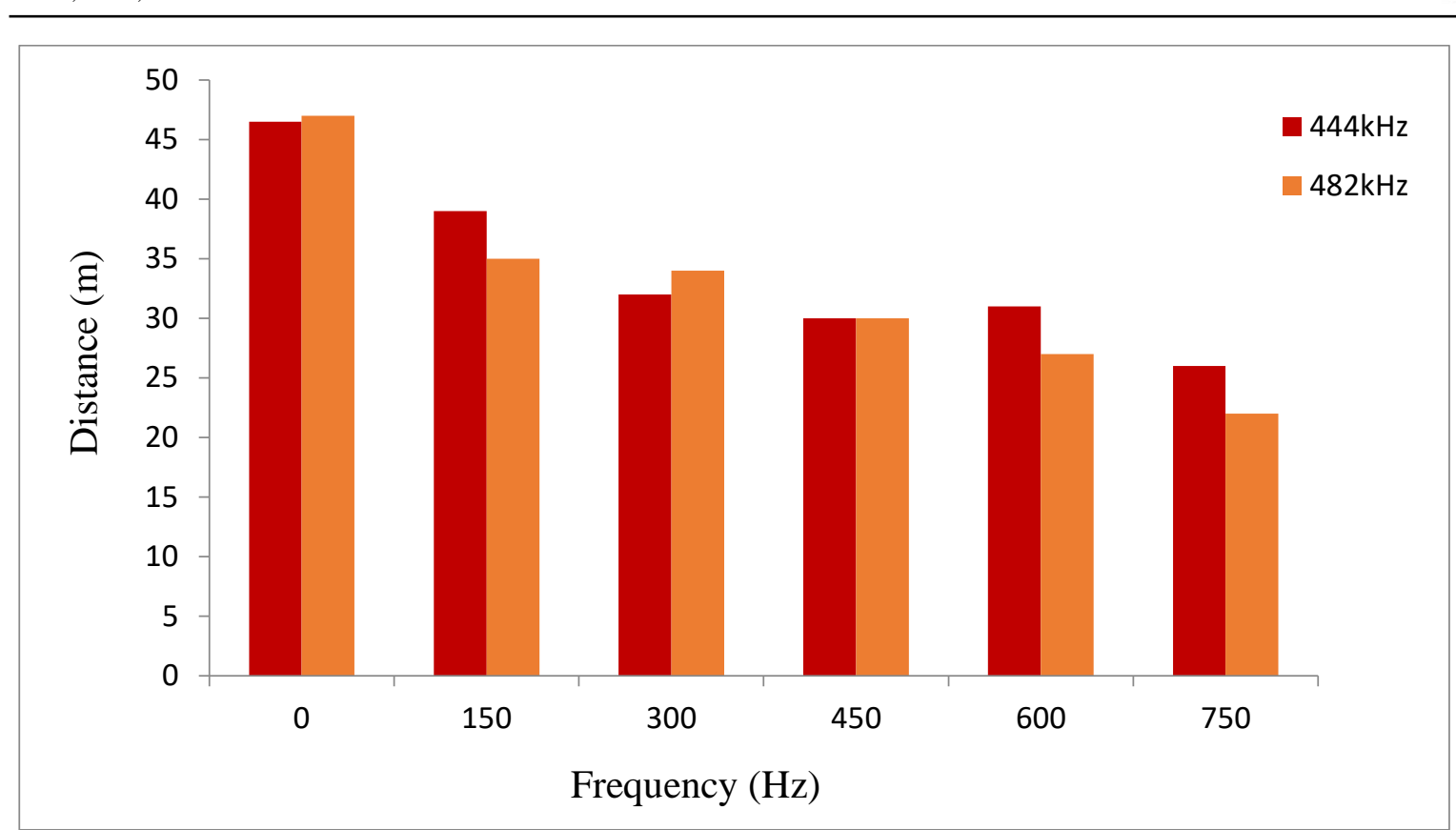

Figure 3: Distance away from power line at frequencies of $444 \mathrm{kHz}$ and $482 \mathrm{kHz}$

\subsection{Conclusion}

This study has analyzed the impact of signal radiation leakage on power line communication system with the aim of determining the conditions for good performance of the PLC system so as to suggest possible means of ensuring improvements in the power line communication system. Power line communication is an effective means of communication in areas where the installation and maintenance of the communication is costly.

The study comprises both theoretical and experimental analysis. The experimental work was conducted in the form of Field measurements, performed in real power line communication network. For the theoretical work, PLC networks were modeled and simulated with the Numerical Electromagnetic Code (NEC).

The results of the study showed that the frequencies of power line operation lie between $444 \mathrm{kHz}$ and $482 \mathrm{kHz}$. This inicated that as wire spacing of powerlines increases, the external field cancellation degrades which results in higher radiation leakages. In general the PLC electric field along and near the power line decay with distance from the device but in at least one case, the electric field increased with increasing distance from the PLC device. Therefore, PLC should be used because it reduces stress in terms of smart grid, numerical electromagnetic code and field measurement.

PLC is a promising emerging technology, which has attracted much attention due to the wide availability of power distribution lines. The result also indicate that PLC is an important alternative to be considered for providing broadband access to remote areas. PLC is cost effective, a permanent solution for transmission of information and data, feasible than other relevent techniques. PLC play a key role in the development of rural area by providing basic voice, market prices and information. 


\subsection{References}

[1] Ajibade, A. O., Oluwafemi, I. B., Ojo, A. O. and Adeniji, K. A. (2017). Theoretical analysis of transmission parameters and interference issues in power line communication systems. ABUAD Journal of Engineering Research and Development (AJERD) 1(1): 95-99.

[2] Anju, K. N, Shyju, Y. (2016). A study in integrated system of power line communication and visible light communication. International Conference on Emerging Trends, 1-14.

[3] Arshad, J. and Shubhangi, A. (2015). Secure power line communication: Review International Journal of advanced computational engineering and networking. 13(12): 13-16.

[4] Berger, L. T., Andreas S. and Escudero-Garzás, J. J. (2012). Power line communications for smart grid applications. Hindawi Publishing, Journal of Electrical and Computer Engineering, 2 013, Article ID 712 376:1-17.

[5] Bhimsen, R. and Noorianah, L. (2017). Modeling of power line communications for indoor system using MIMO coding scheme. International Journal of Engineering Science Invention, 6(12): 35-47.

[6] Darware, A., Azeema, N., Tejani, A., Tejani, S. and Bisane, J. (2012). The role of power line communication in rural area. International Journal of Networking, 2(1): 4449.

[7] Edward, P. G., Julián, J. I., and Cesar, Y. B. (2008). Throughput analysis over power line communication channel in an electric noisy scenario. International Journal of Electronics and Communication Engineering, 2(7): 1344-1350.

[8] Franek, L. and Fiedler, P. (2017). A multi-conductor model of power line communication in medium-voltage lines. Energies Journal, 10, 816; doi:10.3390/en10060816; 1-16.

[9] Luis, G. S., Costa, A. C. M.. De Queiroz, B. A., Vinicius, I. R. Da Costa, T. and Moises V. R. (2017). Coupling for power line communication: a survey, 32(1): 1-13.

[10] Jero, A. (2003). Applicability of power-line communications to data transfer of on-line condition monitoring of electrical drives. Ph.D Thesis submitted to the Lappeenranta University of Technology, Lappeenranta, Finland; 1-141.

[11] Mehdi, K. (2012). Power line communication channel modelling and performance evaluation. Master thesis submitted to the Faculty of Engineering and Industrial Sciences Swinburne University of Technology, 1-147.

[12] Mickey D. and Cox, N. J. (2016). Champagne. near-field coupling between Broadband over Power Line (BPL) and high-frequency communication systems. Mridula Karduri, IEEE Transactions on Power Delivery, 21(4): 2-13.

[13] Modisa, M. (2014). Power line communication (PLC) channel measurements and charaterization. Master Dissertation Submitted to the Department of Electronic Engineering University of Kwazulu-Natal; 1-88.

[14] Ntia Report (2004). Potential interference from Broadband over Power Line (BPL) systems to federal government radio communications at $1.780 \mathrm{mhz}$ phase 1, 1: 04413.

[15] Parhyar, N. R., Shah, M. A. and Lodro, M. M. (2015). Simulation and mathematical modelling of power line communication channel for high data transfer rate. Quaid-EAwam University Research Journal of Engineering, Science and Technology, 14(2): 13-19. 
[16] Rönnberg, S. (2011). Power line communication and customer equipment. Master Thesis submitted to the Luleå University of Technology Department of engineering Sciences and Mathematics Division of Energy Engineering. 1-96.

[17] Sagar, N. (2011). Power line communications systems: overview and analysis. Master thesis submitted to the Graduate School-New Brunswick Rutgers, The State University of New Jersey, 1-160.

[18] Sekyere, F. (2009). Signal radiation leakage of power line communication systems. Master Thesis submitted to the Department of Telecommunication Engineering, Kwame Nkrumah University of Science and Technology, 1-65.

[19] Virginie, D., Pierre, L, Martine, L. and Pierre, D. (2011). Compromising radiated emission from a power line communication cable. Journal of Communications Software and Systems, 7(1), 16-21.

[20] Vivek, A, Nitin, P. and Ankush, D. (2014). Power line communication systems. International Journal of Innovative Research in Electrical, Electronics, Instrumentation and Control Engineering, 2(1): 2-17.

[21] Werner, S. M., Rodger, C. J. and Thomson, N. R. (2005). Identifying power line harmonic radiation from an electrical network. Annales Geophysicae, 23, 2107-2116, 2005 SRef-ID: 1432-0576/ag/2005-23: 2107-2116.

[22] Xiao, C., Jie, F, Kaining, L., Yongxian, Y. and Faye, Z. (2017). Transmission characteristics of high frequency signal in low voltage power lines. International Journal of Information and Communication Sciences, 2(6), 104-109. 